\title{
Metamaterials for surface waves
}

\author{
S. ENOCH
}

\author{
Institut Fresnel, CNRS, Marseille France
}




\section{Metamaterials for surface waves.}

\section{S. ENOCH}

Institut Fresnel, CNRS, Marseille France.
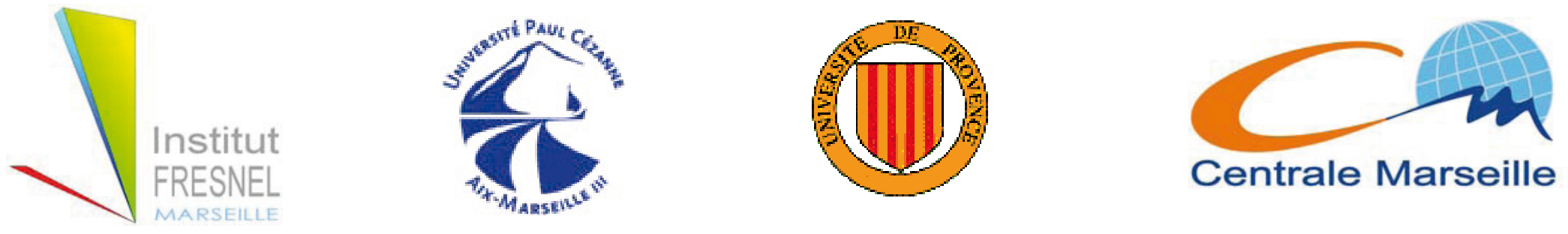


\section{Introduction}

\section{Institut Fresnel}

Photonics, Electromagnetism, Image and Signal Processing. about 60 permanent researchers (universities, engineering school, and cnrs) and 140 people overall. www.fresnel.fr

In the city where the Fabry-Perot interferometer was invented

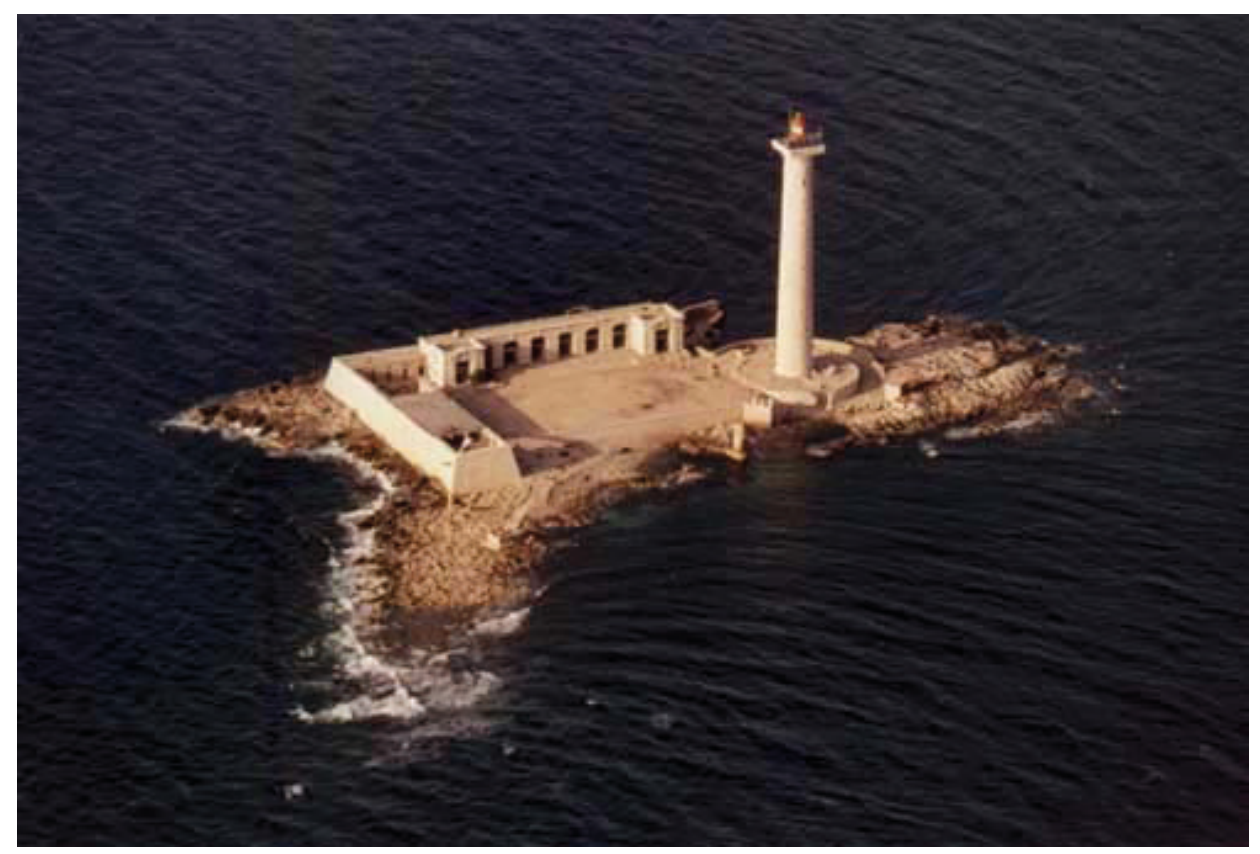

Planier lighthouse in Marseille

Construction supervised by A. Fresnel 


\title{
Introduction
}

Collaborators

PhD students: M. Farhat, M. Kadic, G. Dupont, CNRS researcher: S. Guenneau.

\author{
and also
}

A. B. Movchan, Liverpool University (water waves and thin plates)

R. Quidant's group, ICFO, Barcelona (plasmonics) 


\section{Cloaking: our motivations}

- Looking for experiments for popularization of the metamamaterial's concepts.

- $\quad$ Promote the metamaterials ideas for other type of waves (water waves, flexural waves, surface plasmons polaritons). 


\section{Cloaking by transformation (reminder)}

J.B. Pendry, D. Schurig, \& D. R. Smith, Science 312, 1780-1782 (2006).

Coordinates transform (2D case)

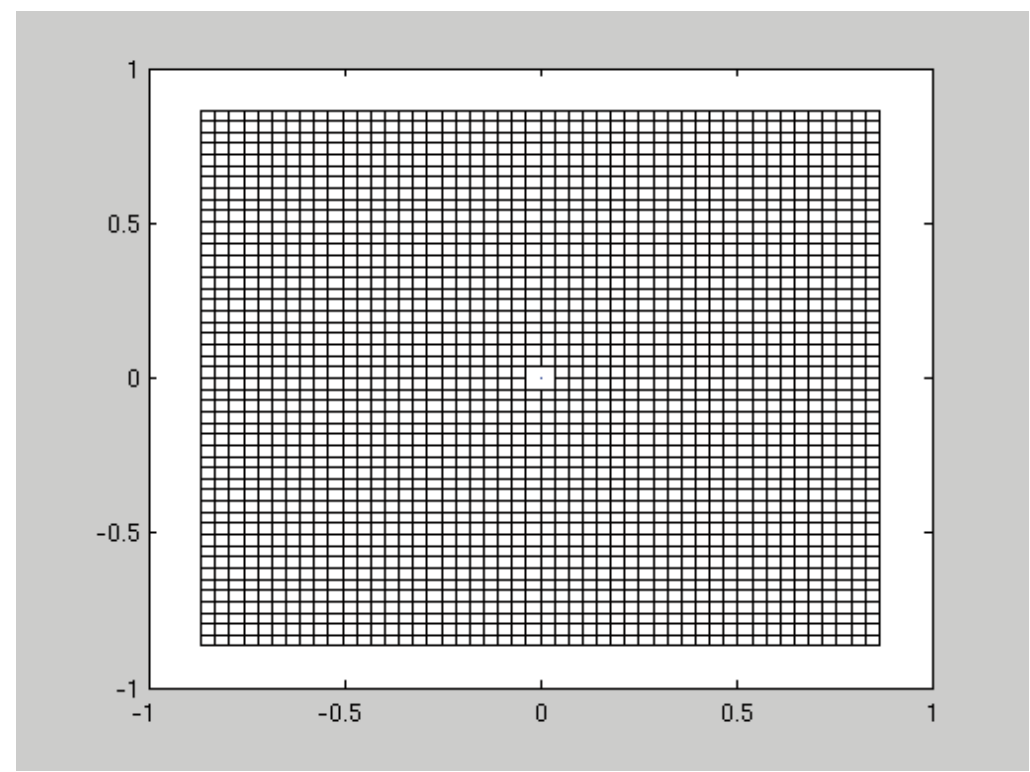

$$
\begin{aligned}
& r^{\prime}=R_{1}+r\left(R_{2}-R_{1}\right) / R_{2} \\
& \theta^{\prime}=\theta \text { and } x_{3}^{\prime}=x_{3}
\end{aligned}
$$

Permeability and permittivity

$$
\varepsilon_{r}=\mu_{r}=\frac{r-R_{1}}{r}
$$

$$
\varepsilon_{\theta}=\mu_{\theta}=\frac{r}{r-R_{1}}
$$

$$
\varepsilon_{3}=\mu_{3}=\left(\frac{R_{2}}{R_{2}-R_{1}}\right)^{2} \frac{r-R_{1}}{r}
$$




\section{Cloaking by transformation}

J.B. Pendry, D. Schurig, \& D. R. Smith, Science 312, 1780-1782 (2006).

Coordinates transform

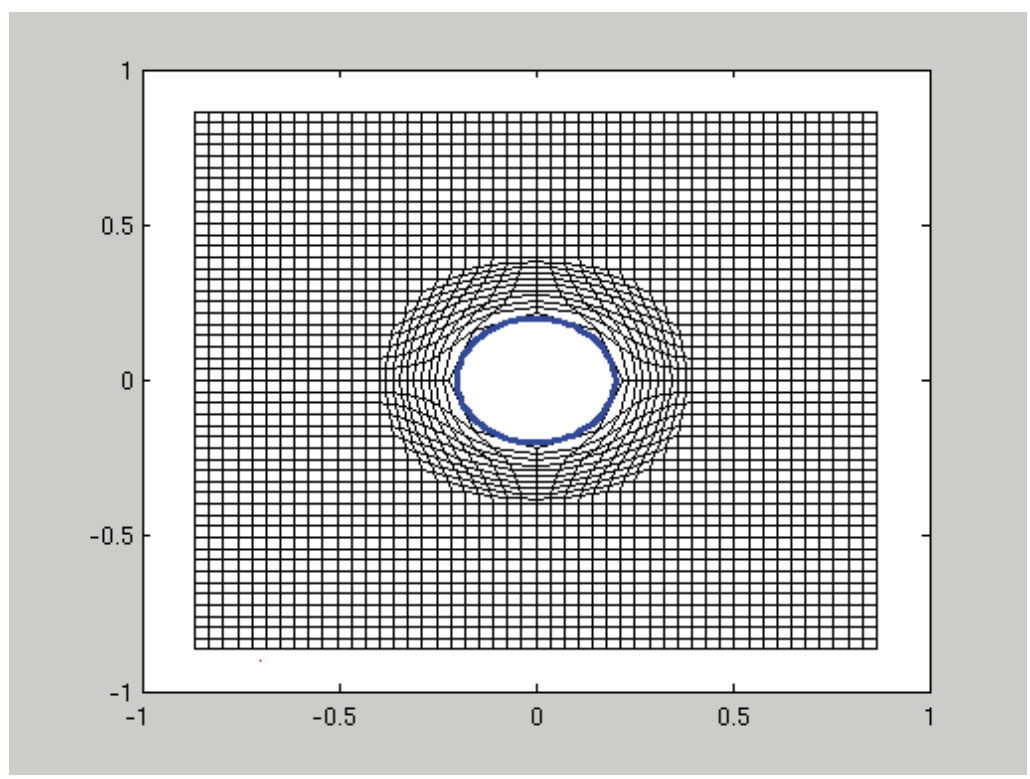

$$
\begin{aligned}
& r^{\prime}=R_{1}+r\left(R_{2}-R_{1}\right) / R_{2} \\
& \theta^{\prime}=\theta \text { and } x_{3}^{\prime}=x_{3}
\end{aligned}
$$

Permeability and permittivity

$$
\varepsilon_{r}=\mu_{r}=\frac{r-R_{1}}{r} \quad \varepsilon_{\theta}=\mu_{\theta}=\frac{r}{r-R_{1}} \quad \varepsilon_{3}=\mu_{3}=\left(\frac{R_{2}}{R_{2}-R_{1}}\right)^{2} \frac{r-R_{1}}{r}
$$




\section{Cloaking by transformation}

Remark : « Nearly » invisible in TE polarization (magnetic field perpendicular to the figure):

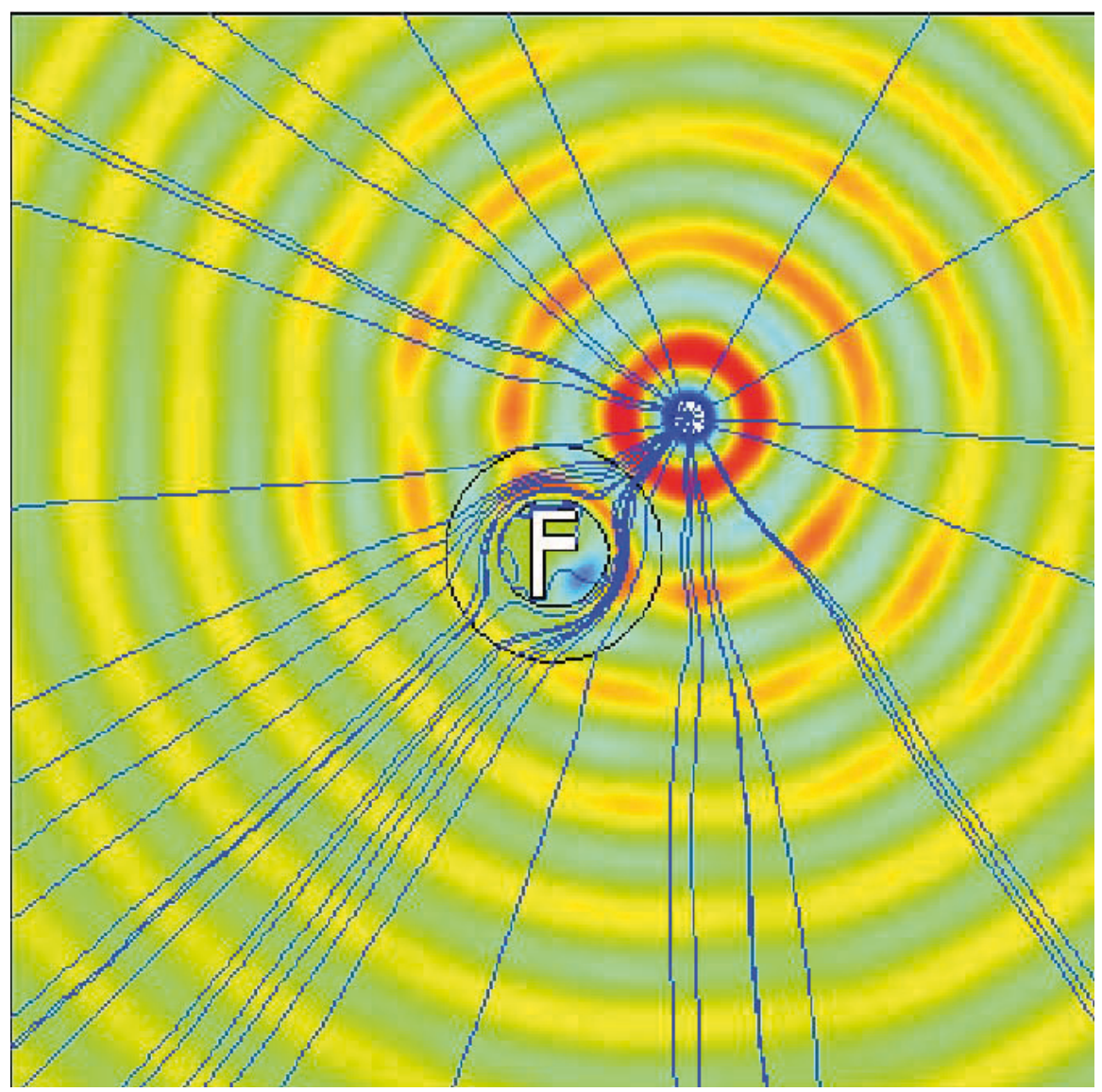

$$
\begin{gathered}
\varepsilon_{r}=\left(\frac{R_{2}}{R_{2}-R_{1}}\right)^{2}\left(\frac{r-R_{1}}{r}\right)^{2} \\
\varepsilon_{\theta}=\left(\frac{R_{2}}{R_{2}-R_{1}}\right)^{2} \\
\mu_{3}=1
\end{gathered}
$$




\section{Other transformations}

Concentrator

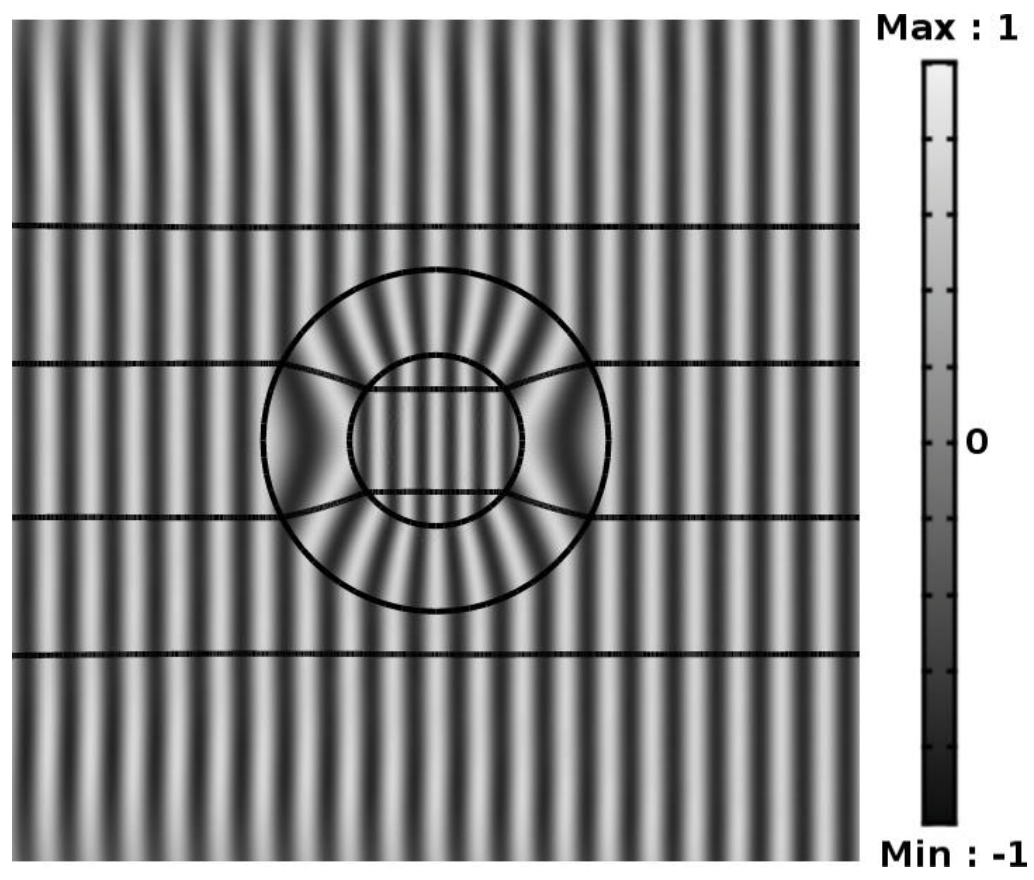

A plane wave at wavelength $\lambda=0.2$ on a concentrator with inner radius $\mathrm{R} 1=0.2$, 'virtual' radius $\mathrm{R} 2=0.3$ and outer radius $\mathrm{R} 3=0.4$; We note that the wavelength of the field inside the disc is shrunk, which is the concentrating effect, but the device is perfectly invisible. 


\section{Other transformations}

Arbitrary shaped invisibility cloak

Arbitrary shaped invisibility cloak in presence of a line source of wavelength $\lambda=0.3$ located at $(x, y)=(1,-1)$;

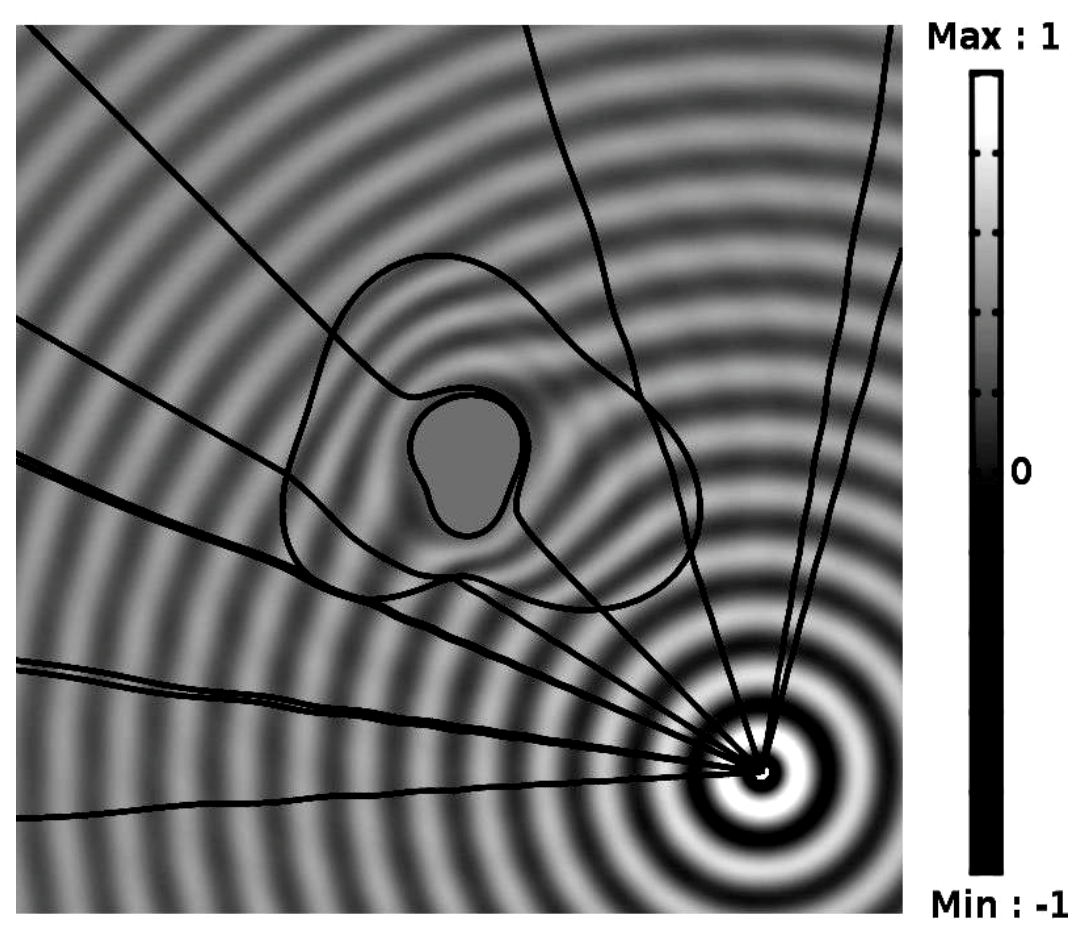




\section{From Navier-Stokes to Helmholtz}

Let $\mathrm{u}\left(\mathrm{x}_{1} ; \mathrm{x}_{2} ; \mathrm{x}_{3} ; \mathrm{t}\right)$ satisfy the Navier-Stokes equation :

$$
\frac{\partial \mathbf{u}}{\partial t}+\mathbf{u} \cdot \nabla \mathbf{u}+\frac{\nabla p}{\rho}=g+v \Delta \mathbf{u}
$$

Neglecting the liquid viscosity and assuming an incompressible and irrotational liquid flow and thus noting $\mathbf{u}=\nabla \phi$, we obtain :

$$
\nabla^{2} \Phi=0
$$

BUT : Laplace equation manifests no wave character ! 


\section{From Navier-Stokes to Helmholtz}

$\rightarrow$ Waves are induced by the boundary conditions on the free surface air-liquid.

Indeed, neglecting surface tension, we obtain on the free surface :

$$
\frac{\partial \Phi}{\partial t}+\frac{|\nabla \Phi|}{2}+\frac{p_{0}}{\rho}+g \xi=f(t), \text { on } x_{3}=\xi
$$

For a harmonic potential with frequency $\omega$, we can choose the following form of $\Phi$ :

$$
\Phi\left(x_{1}, x_{2}, x_{3}, t\right)=\Re e\left(\phi\left(x_{1}, x_{2}\right) \cosh \left(\kappa x_{3}\right) e^{-i \omega t}\right)
$$

$\kappa$ is the spectral parameter and $\phi$ the reduced potential, solution of the 2D Helmholtz equation on a free surface. 


\section{From Navier-Stokes to Helmholtz}

2D Helmholtz equation on a free surface

$$
\nabla^{2} \phi+\kappa^{2} \phi=0
$$

Furthermore, $\kappa$ is linked to the wave-frequency $\omega$ via the relation :

$$
\omega^{2}=g \kappa\left(1+d_{c}^{2} \kappa^{2}\right) \tanh (\kappa h)
$$

involving the depth of liquid $h$, its capillarity $d_{c}$ and the gravity $g$.

On a rigid obstacle, the boundary condition is a Neuman condition. 


\section{Cloaking Linear Water Waves}

The viscosity matrix is such that the liquid will flow faster in the azimutal direction and will be bent around the central region of the cloak.

$\rightarrow$ Goal : homogenization of the structured cloak to obtain an effective anisotropic fluid.

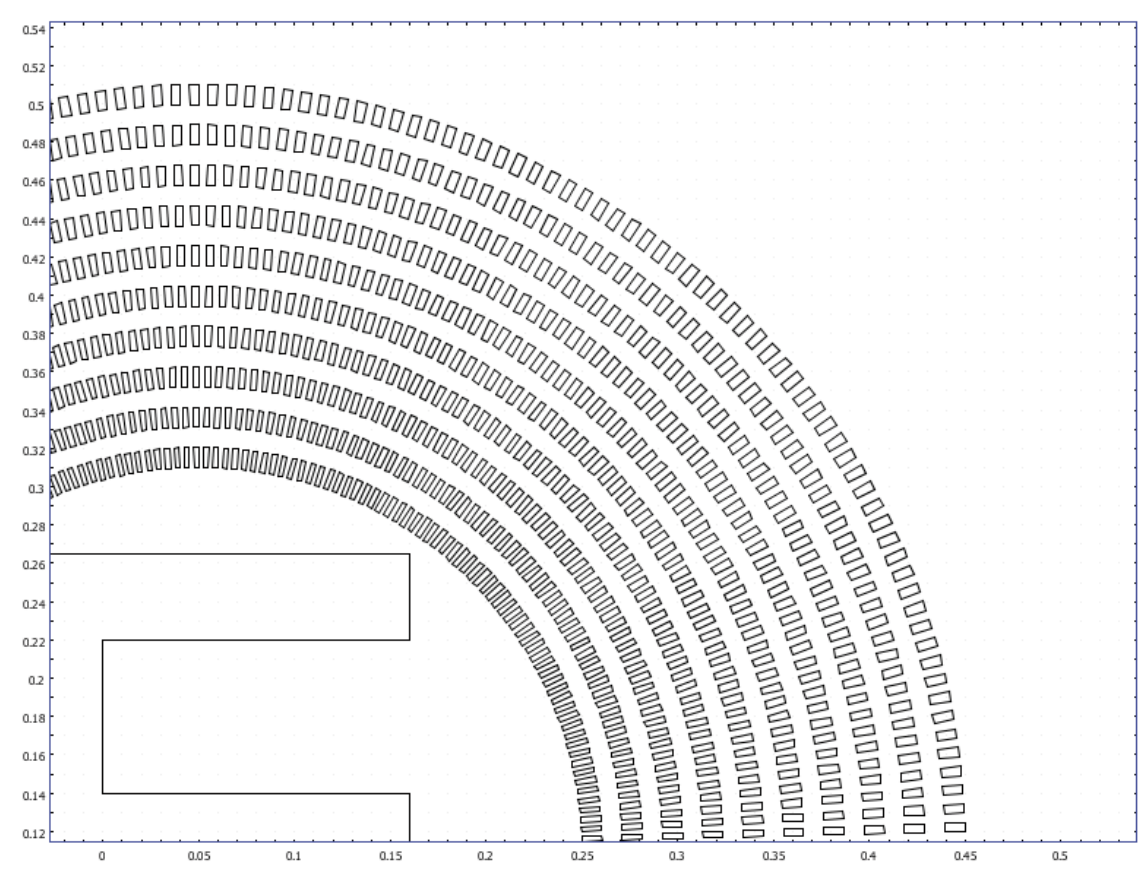




\section{Cloaking Linear Water Waves}

Taking the two-scale limit in the fast-oscillating potential field, we obtain the following homogenized problem when $\eta \rightarrow 0$ :

$$
\nabla\left(\left[\mu_{\text {hom }}\right] \nabla \phi_{\text {hom }}(\mathbf{x})\right)=\kappa^{2} \phi_{\text {hom }}(\mathbf{x})
$$

The fluid flowing within the homogenized cloak is characterized by the shear viscosity matrix :

$$
\left[\mu_{\text {hom }}\right]=\left[\begin{array}{cc}
1.7 & 0 \\
0 & 8.2
\end{array}\right]
$$




\section{Cloaking Linear Water Waves}
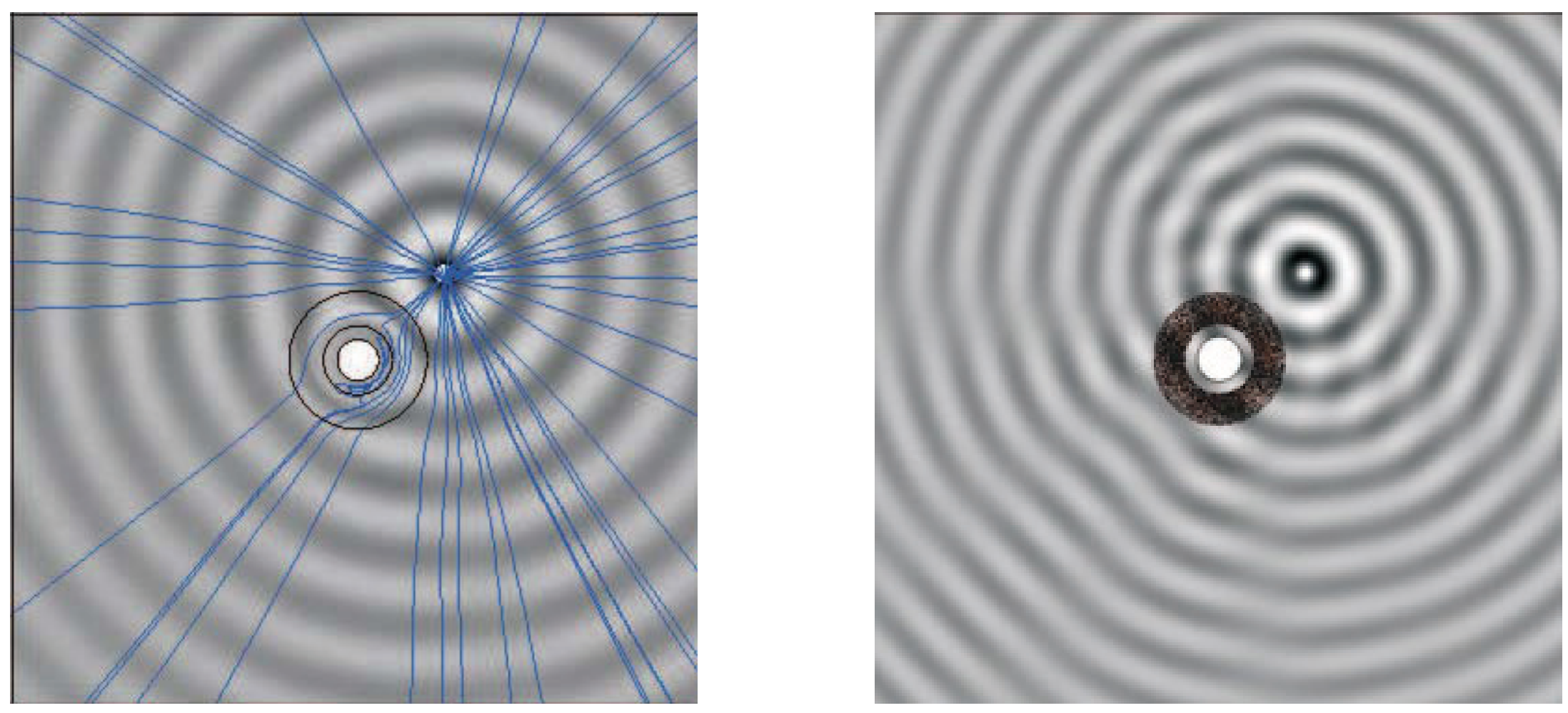


\section{Cloaking Linear Water Waves}

A classical experimental setup used in secondary schools:
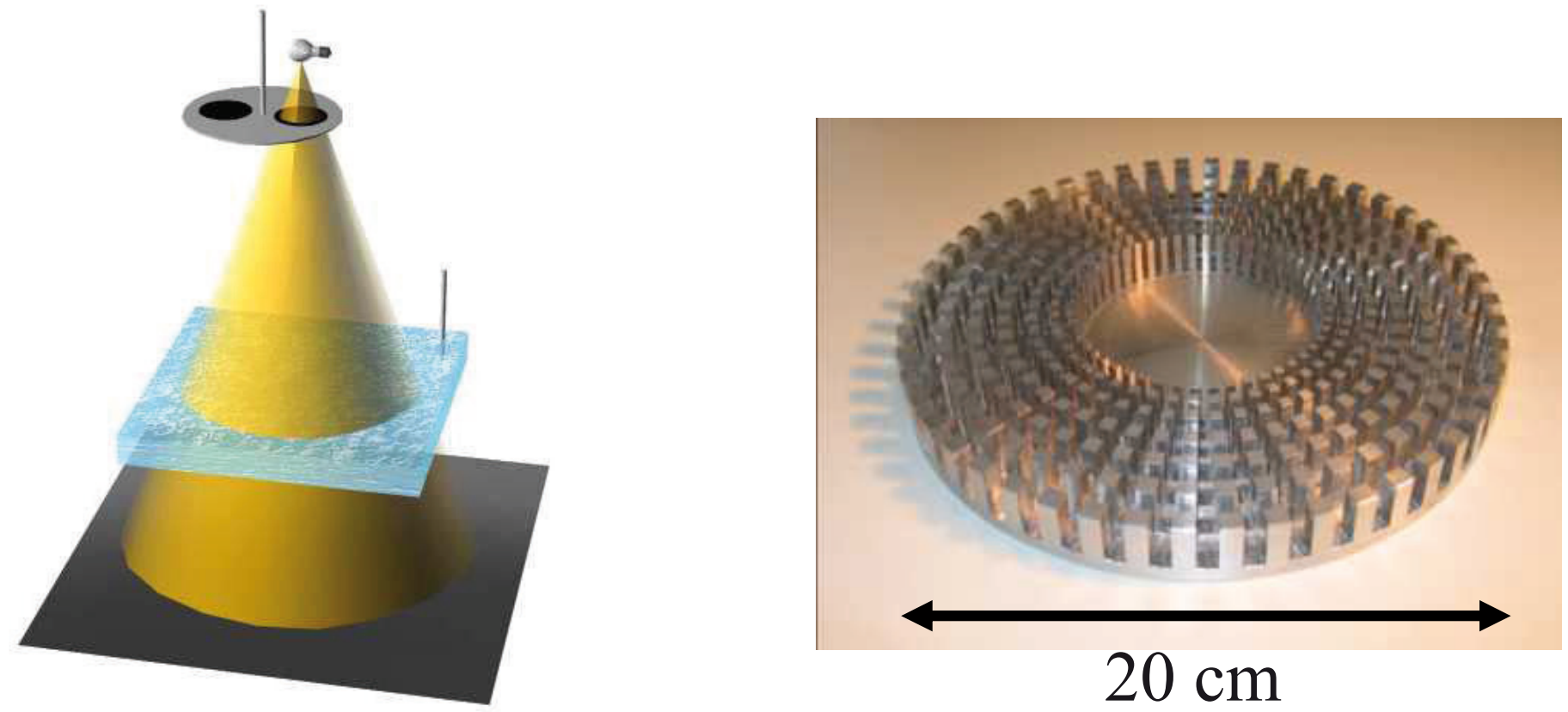

M. Farhat, S. Enoch, S. Guenneau, and A. B. Movchan, "Broadband cylindrical acoustic cloak for linear surface waves in a fluid", Physical Review Letters 101,.134501 (2008). 


\section{Cloaking Linear Water Waves}

Methoxynonafluorobutane: viscosity $0,61 \mathrm{~mm}^{2} / \mathrm{s}$ density $1,529 \mathrm{~g} / \mathrm{mL}$.
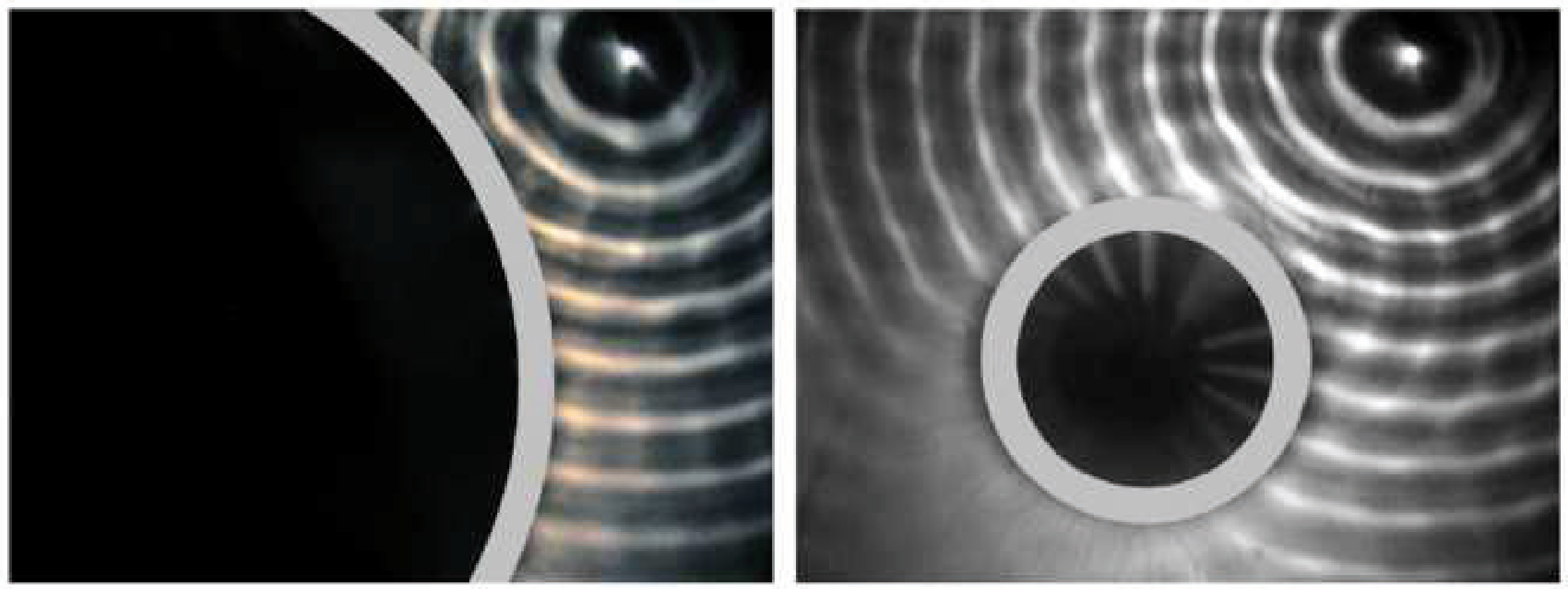


\section{Thin Elastic Plates}

Transformation Electrodynamics is still challenging but ...

The wavelength $\lambda$ is supposed to be large enough compared to the thickness of the plate $h$ and small compared to its in-plane dimension $L$, i.e. $h<<\lambda<<L$.

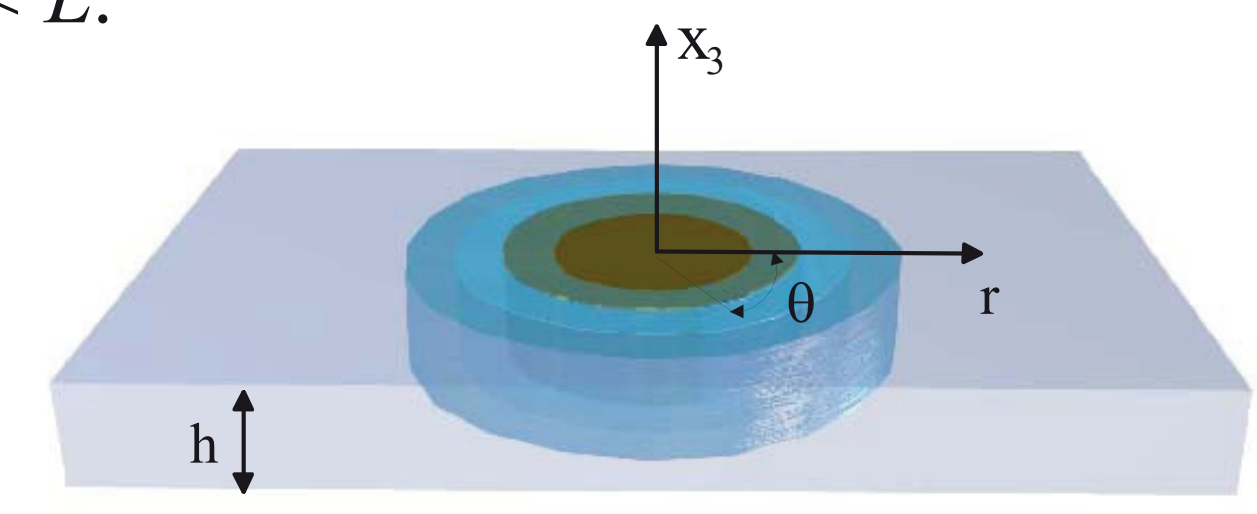

The out-of-plane displacement $\mathrm{u}=(0 ; 0 ; U(r ; \theta))$ in the $x_{3}$-direction (along the vertical axis) is solution of:

$$
\langle\lambda\rangle \nabla \cdot\left(\underline{\underline{\zeta}}^{-1} \nabla\left(\langle\lambda\rangle \nabla \cdot\left(\underline{\underline{\zeta}}^{-1} \nabla U\right)\right)\right)-\beta_{0}^{4} U=0
$$




\section{Thin Elastic Plates}

(a)

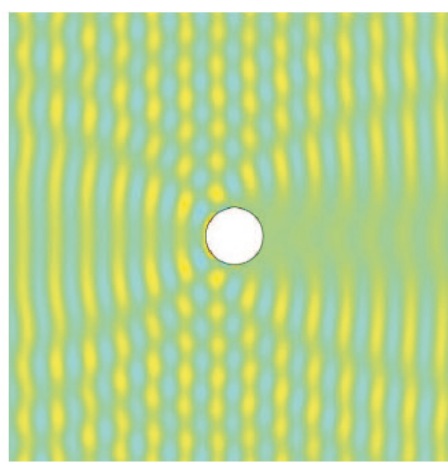

(c)

a rigid clamped obstacle surrounded by a multilayered isotropic cloak (inner radius $a=$ $0.3 \mathrm{~m}$, outer radius $b=0.6 \mathrm{~m}$ and 20 layers, for an incoming plane wave of frequency $22.5 \mathrm{~Hz}$
Left: Real part of the displacement scattered by a rigid clamped obstacle of radius $0: 25 \mathrm{~m}$ for an incoming plane wave of frequency $22: 5 \mathrm{~Hz}$

Right: Real part of the displacement scattered by (b)

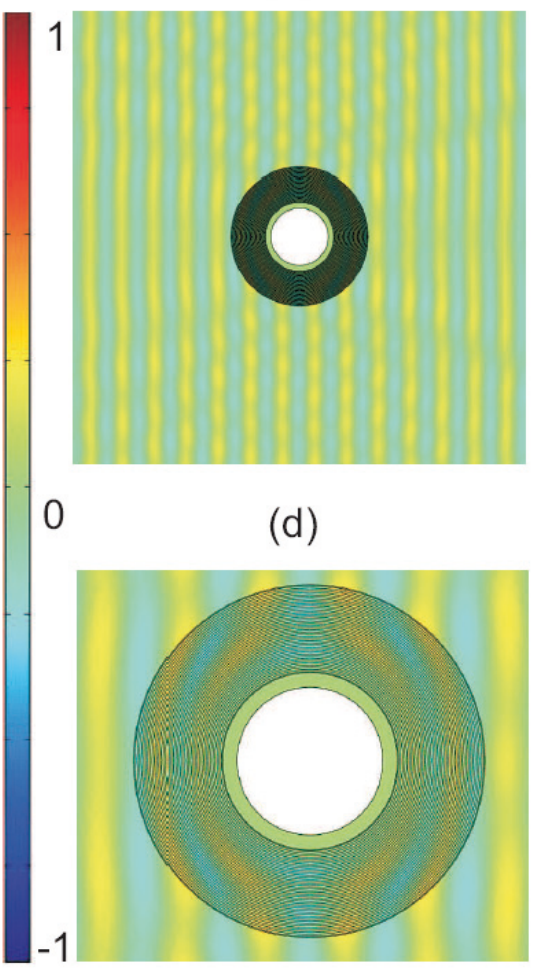

M. Farhat, S. Guenneau, S. Enoch, A. B. Movchan, "Cloaking bending waves propagating in thin elastic plates," Physical Review B 79, 033102 (2009).

M. Farhat, S. Guenneau, and S. Enoch, "Ultrabroadband Elastic Cloaking in Thin Plates", Physical Review Letters 103, 024301 (2009) 


\section{Plasmonic Carpet}

We consider the following transformation

$$
\left\{\begin{array}{l}
x^{\prime}=\frac{x_{2}(y)-x_{1}(y)}{x_{2}(y)} x+x_{1}(y), 0<x<x_{2}(y), \\
y^{\prime}=a<y<b, \\
z^{\prime}=z, 0<z<+\infty
\end{array}\right.
$$

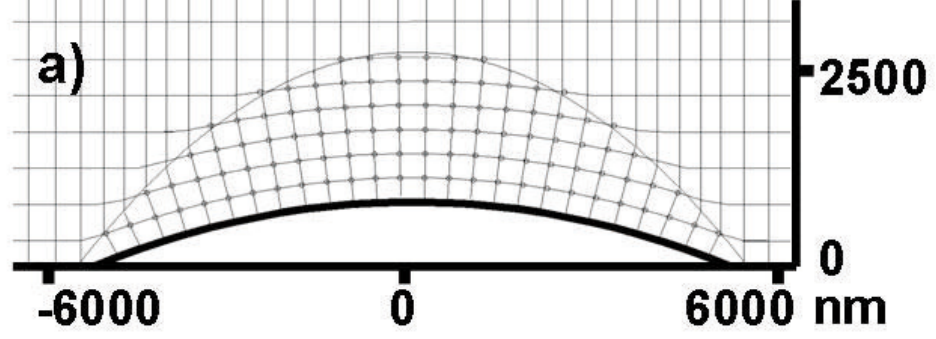

That gives us the permittivity and permeability tensors.

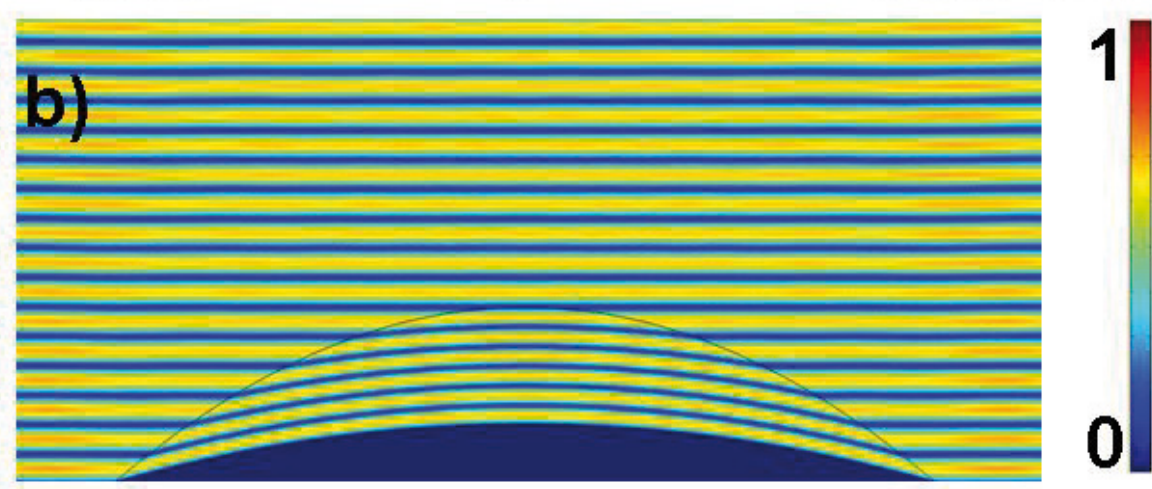

J. Li, and J. B. Pendry, "Hiding under the carpet: a new strategy for cloaking," Phys. Rev. Lett. 101(20), 203901(2008). 


\section{Plasmonic Carpet}
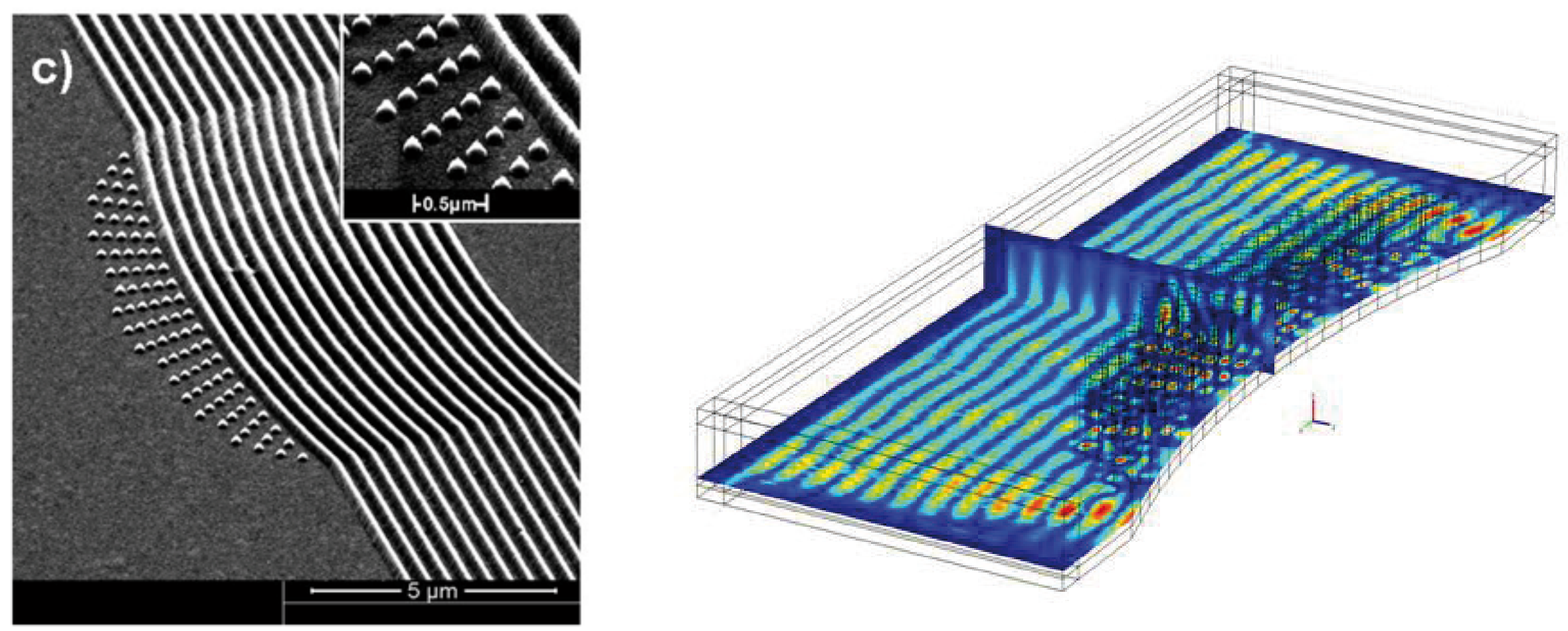

We chose a configuration in which a gold surface is structured with $\mathrm{TiO}_{2}$ nanostructures.

The shape of the obtained TiO2 particles is conical ( $h=200 \mathrm{~nm}, \mathrm{r}=210 \mathrm{~nm}$ ) as a consequence of the etching anisotropy. 


\section{Plasmonic Carpet}

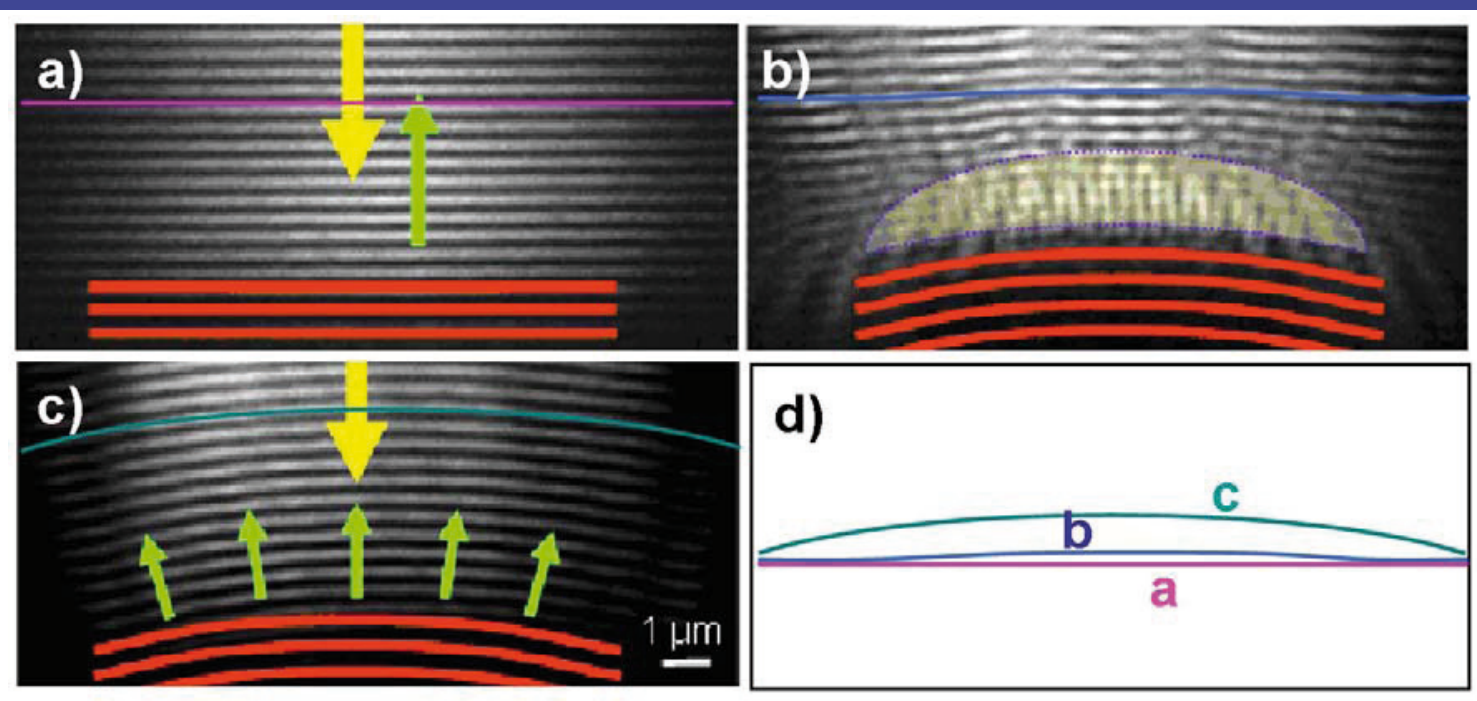

Comparing the areas under the numerically averaged curves $b$ (curved mirror with carpet) and c (curved mirror without carpet) leads to reduction by a factor 3.7.

J. Renger, M. Kadic, G. Dupont, S. S. Aćimović, S. Guenneau, R. Quidant, S. Enoch, "hidden progress: broadband plasmonic invisibility" optics express 18, 1575715768 (2010).

P. A. Huidobro, M. L. Nesterov, L. Martin-Moreno, and F. J. García-Vidal, "Transformation Optics for Plasmonics," http://arxiv.org/abs/1003.1154.

Y. Liu, T. Zentgraf, G. Bartal, and X. Zhang, "Transformational Plasmon Optics," http://arxiv.org/abs/1003.1326. 


\section{Conclusion}

- Larger scale Nonlinearity

Collaboration with B. Molin (IRPHE, Marseille)

- Applications : anti vibration systems, fish farming...

- Apply metamaterials concepts to other type of waves.

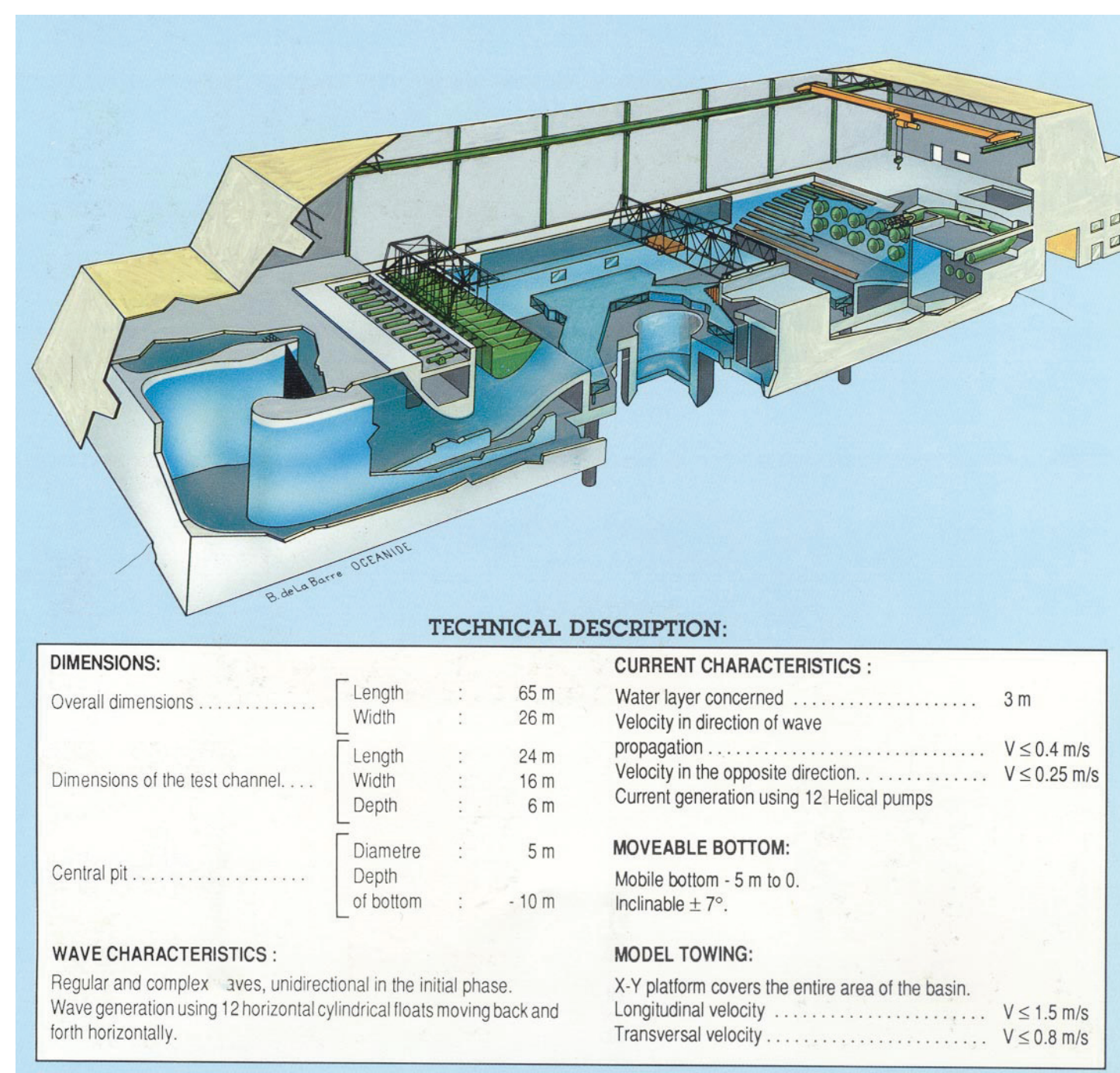

BGO FIRST, La Seyne sur Mer 